\title{
ANALYSIS AND MODELING OF A TWO-PHASE JET PUMP OF A THERMLL MANAGEMENT SYSTEM FOR AEROSPACE APPLICATIONS
}

\author{
S.A. Sherif W.E. Lear J.M. Steadham \\ Deparment of Mechanical Engineering \\ University of Florida \\ 237 MEB, P.O. Box 116300 \\ Gainesville, Florida 32611-6300
}

\author{
P.L. Hunt J.B. Holladay \\ Thermal and Life Support Division \\ NASA-Marshall Space Flight Center \\ Huntsville, AL 35812
}

\begin{abstract}
Jet pumps are devices capable of pumping fluids to a higher pressure by inducing the motion of a secondary fluid employing a high speed primary fluid. The main components of a jet pump are a primary nozzle, secondary fluid injectors, a mixing chamber, a throat, and a diffuser. The work described in this paper models the flow of a two-phase primary fluid inducing a secondary liquid (saturated or subcooled) injected into the jet pump mixing chamber. The model is capable of accounting for phase transformations due to compression, expansion, and mixing. The model is also capable of incorporating the effects of the temperature-and pressure-dependency in the analysis. The approach adopted utilizes an isentropic constantpressure mixing in the mixing chamber and at times employs iterative techniques to determine the flow conditions in the different parts of the jet pump.
\end{abstract}

\section{NOMENCLATURE}

\section{Latin Symbols:}

\footnotetext{
A cross-sectional area, $\mathrm{m}^{2}$

a speed of sound, $\mathrm{m} / \mathrm{s}$

h specific enthalpy, $\mathrm{kJ} / \mathrm{kg}$

M Mach number, dimensionless

m mass flow rate, $\mathrm{kg} / \mathrm{s}$

$\mathrm{P}$ pressure, $\mathrm{kPa}$

s $\quad$ specific entropy, $\mathrm{kJ} / \mathrm{kg} . \mathrm{K}$

$\mathrm{T}$ temperature, ${ }^{\circ} \mathrm{C}$

Copynght ${ }^{\circ} 1998$ by S.A. Shenf. Pudisted by AIAA with permussion.
}

$\begin{array}{ll}\mathrm{V} & \text { velocity, } \mathrm{m} / \mathrm{s} \\ \mathrm{x} & \text { quality }\end{array}$

\section{Greek Svmbols:}

$\Phi$ entrainment ratio, $\dot{\mathrm{m}}_{\mathrm{s}} / \dot{\mathrm{m}}_{\mathrm{p}}$, dimensionless $\rho$ density, $\mathrm{kg} / \mathrm{m}^{3}$

\section{Subscripts:}

$\begin{array}{ll}\text { d } & \text { diffuser } \\ \text { de } & \text { diffuser exit } \\ \text { e } & \text { exit } \\ \text { f } & \text { saturated liquid condition } \\ \text { g } & \text { saturated vapor condition } \\ \text { I } & \text { inlet } \\ \text { m } & \text { mixture } \\ \text { me } & \text { mixing chamber exit } \\ \text { mi } & \text { mixing chamber inlet } \\ \text { n } & \text { primary nozzle } \\ \text { ne } & \text { primary nozzle exit } \\ \text { ni } & \text { primary nozzle inlet } \\ \text { o } & \text { stagnation condition } \\ \text { p } & \text { primary fluid } \\ \text { s } & \text { secondary fluid } \\ \text { se } & \text { secondary fluid exit } \\ \text { si } & \text { secondary fluid inlet } \\ \text { ss } & \text { conditions downstrean of shock wave }\end{array}$




\section{NTRODLCTION}

Jet pumps are devices capable of pumping tluids to a higher pressure employing a nozzle/diffuser/mixing chamber combination. A primary fluid is usually allowed to pass through a converging-diverging nozzle where it can accelerate to supersonic speeds at the nozzle exit. The relatively high kinetic energy that the primary fluid possesses at the nozzle exit is accompanied by a low pressure region in order to satisfy momentum conservation requirements. The low pressure region downstream of the nozzle exit permits a secondary fluid to be entrained into and mixed with the primary fluid in a mixing chamber located downstream of the nozzle. Several combinations may exist in terms of the nature of the primary and secondary fluids in so far as whether they are single- or two-phase fluids. Depending on this, the jet pump may be classified as gas/gas, gas/liquid, liquid/liquid, two-phase/liquid, or other similar combinations.

The mixing chamber serves to create a homogeneous single-phase or two-phase mixture which is ready to enter a diffuser where the high kinetic energy of the mixture is converted into pressure energy. If the fluid mixture entering the diffuser is in the supersonic flow regime, a normal shock wave may develop inside the diffuser. If the fluid mixture is one that can easily undergo phase transformations, a condensation shock may develop. Because of the overall rise in pressure in the diffuser as well as the additional rise in pressure caused by the shock wave, condensation of the fluid mixture becomes more likely. If the two-phase flow entering the diffuser is predominantly gaseous with liquid droplets suspended in it, chances are that it would transform into a predominantly liquid flow containing gaseous bubbles (bubbly flow).

Because of the high speed of the primary fluid, there exists a region known as the potential core region where entrainment and mixing between the primary and secondary fluids does not take place. Thus, as the primary fluid enters the mixing section and expands, and because of the lack of mixing in the potential core region, the peripheral annular area available for the secondary fluid will shrink. Because of continuity considerations, the secondary fluid will accelerate in the annular region and it would be conceivable that it reaches sonic speed there. This secondary fluid acceleration will be accompanied by a reduction in pressure in the peripheral area which would in tum make it more likely for the otherwise subcooled or saturated secondary fluid to flash into vapor, thus forming either a two-phase mixture or pure vapor in the region occupied by the secondary fluid. Now that the secondary fluid may have become predominantly or purely vapor, any further motion downstream in the region occupied by the secondary flow is likely to be in the supersonic regime and might be accompanied by shock waves in the peripheral region. As the potential core region ends, the now two-phase or pure vapor secondary fluid will start a mode of mixing with the already two-phase primary fluid in the remainder of the mixing section downstream of the potential core region and will form a homogeneous two-phase mixture which is bound towards the diffuser. As stated earlier, this two-phase mixture will undergo a velocity reduction accompanied by a pressure rise and will be subject to undergoing a shock wave somewhere in the diffuser.

Literature dealing with jet pumps is abundant and covers a very wide array of application areas. Example application areas includes vacuum pumps which are used in the food industry, power station work, and the chemical industry; ejector systems which have applications in the aircraft industry as cabin ventilators and for purposes of jet thrust augmentation; jet pumps which are used in the oil industry for oil well pumping; and steam-jet ejector refrigeration, to just name a few. Examples of work relevant to this investigation includes those of Holmes et al. (1987). Cunningham and Dopkin (1974), Elger et al. (1991). Marini et al. (1992), Jiao et al. (1990), Neve (1991), Bredikhin et al. (1990), and Fairuzov and Bredikhin (1995).

One of the primary motivations behind this work is to attempt to understand the performance of a two-phase jet pump which constitutes part of a flow boiling test facility at NASA-Marshall. The flow boiling apparatus is intended to provide data necessary to design highly efficient two-phase thermal control systems for aerospace applications. The facility will also be capable of testing altemative refrigerants and evaluate their performance using various heat exchangers with enhanced surfaces. The test facility is also intended for use in evaluating single-phase performance of systems currently using CFC refrigerants (Holladay and Hunt, 1996). 
While past researchers have been able to model the two-phase flow jet pump using the one-dimensional assumption with no shock waves and no phase change, there is no research known to the authors apart form that of Anand (1992) that was able to account for condensation shocks. The work performed by Fairuzov and Bredikhin (1995), while providing useful insights into the performance of the jet pump under study in this paper, did not fully account for all the issues involved in the constant-pressure mixing process performed in the mixing chamber. Thus, one of the objectives of the work described here is to model the jet pump in question accounting for phase transformations due to expansion, compression, and mixing, and to provide a more accurate description of the constant-pressure mixing process. The analysis employs an isentropic homogeneous expansion/compression model, and while the results are presented for $R-134 a$, the analysis is general enough to be applied to other refrigerants such as R-22 and R-113.

\section{ANALYSIS}

The analysis described in this paper deals with a jet pump whose primary fluid is a two-phase mixture and whose secondary fluid is either a subcooled or saturated liquid having the same chemical composition as the primary fluid. The primary fluid enters a converging-diverging nozzle (primary nozzle) at inlet conditions $P_{n}, T_{n u}$, and $x_{n i}$ for the pressure, temperature, and quality, respectively, and exits at a high velocity and low pressure (see Figure 1). The low pressure at the primary nozzle exit induces the flow of the secondary liquid (which may also enter by the forced action of subcooled liquid spray injectors). The secondary liquid is introduced from an upstream pressure of $\mathrm{P}_{\mathrm{si}}$ (and a low velocity) and enters the mixing section at a pressure of $P_{s e}$. The constantpressure mixing process as modeled in this paper requires the pressure $P_{s c}$ to equal the mixing chamber exit pressure $P_{m e}$. This type of mixing has been selected since it produces compression ratios in the jet pump which are a bit higher than those produced under constant-area mixing conditions.

In order to be able to perform the analysis as stated above, the inlet conditions of both the primary and secondary fluids as well as the entrainment ratio and pump geometry (except the mixing chamber exit area) are assumed known. The mixing chamber exit area, $A_{m e}$, is assumed unknown and will be determined as part of the solution in order to satisfy momentum conservation in the mixing chamber, while imposing the constant-pressure mixing condition. This situation makes the model described here more design (as opposed to analysis) oriented. In what follows, a summary of the equations employed in the model will be presented.

\section{Primary Nozzle:}

The primary nozzle is segmented into two pars for purposes of this analysis; the converging section and the diverging section. In both sections, the isentropic homogenous expansion model is employed. Since the inlet pressure $P_{n i}$, temperature $T_{n}$, and quality $x_{n i}$, are all known, the specific volume, enthalpy, and entropy can be determined:

$$
\begin{aligned}
& v_{n i}=v_{n i, f}+x_{n i}\left(v_{n i, g}-v_{n i, f}\right) \\
& h_{n i}=h_{n i, f}+x_{n i}\left(h_{n i, g}-h_{n i, f}\right) \\
& s_{n i}=s_{n i f f}+x_{n i}\left(s_{n i, g}-s_{n i, f}\right)
\end{aligned}
$$

Applying the continuity and energy equations in the converging section along with the definitions of the Mach number and the speed of sound, the isentropic flow requirement, and the equations of state for the enthalpy and density we get:

$$
\begin{gathered}
\rho_{\mathrm{ni}} \mathrm{A}_{\mathrm{ai}} \mathrm{V}_{\mathrm{ni}}=\rho_{\mathrm{ar}} \mathrm{A}_{\mathrm{at}} \mathrm{V}_{\mathrm{nt}} \\
\mathrm{h}_{\mathrm{ai}}+\frac{1}{2} \mathrm{~V}_{\mathrm{ni}}^{2}=\mathrm{h}_{\mathrm{nt}}+\frac{1}{2} \mathrm{~V}_{\mathrm{nt}}^{2} \\
\mathrm{M}_{\mathrm{at}}=\frac{\mathrm{V}_{\mathrm{nt}}}{\mathrm{a}_{\mathrm{nt}}}=1 \\
\mathrm{a}_{\mathrm{nt}}=\sqrt{\left(\frac{\partial \mathrm{P}}{\partial \rho}\right)_{3}}
\end{gathered}
$$




$$
\begin{aligned}
& s_{\mathrm{at}}=s_{\mathrm{ai}}=s_{\mathrm{ni,f}}+x_{\mathrm{at}}\left(s_{\mathrm{at}, 8}-s_{\mathrm{at}, f}\right)
\end{aligned}
$$

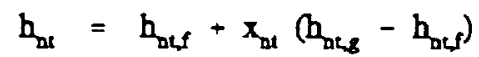

$$
\begin{aligned}
& \frac{1}{\rho_{n t}}=\frac{1}{\rho_{\mathrm{at} f}}+x_{\mathrm{nt}}\left(\frac{1}{\rho_{\mathrm{nug}}}-\frac{1}{\rho_{\mathrm{nt}, f}}\right)
\end{aligned}
$$

The system of equations from Equation (4) through Equation (10) can be solved iteratively by guessing the pressure at the nozzle throat $P_{m}$ and using Equation (8) to compute the quality at the throat $x_{n x}$. The quality can then be used to compute the specific enthalpy and density at the throat using Equations (9) and (10), respectively. Equations (4) and (5) can now be solved in the velocities $V_{n i}$ and $V_{n x}$. The speed of sound at the throat can be computed from Equation (7) and should be compared to the velocity term $V_{n s}$. If the two quantities are found different in value, another value of pressure is tried until convergence.

At this point, the mass flow rate of the primary fluid, $\dot{\mathrm{m}}_{\mathrm{p}}$, may be computed using

$$
\dot{\mathrm{m}}_{\mathrm{p}}=\rho_{\mathrm{nt}} \mathrm{A}_{\mathrm{nt}} \mathrm{V}_{\mathrm{nt}}
$$

The procedure described above can now be repeated between the throat and exit sections of the primary nozzle to produce the quality, density, specific enthalpy, Mach number, and velocity at the exit section. This can be achieved using the following:

$$
\begin{aligned}
& s_{\mathrm{at}}=s_{\mathrm{ne}}=s_{\mathrm{De}, \mathrm{f}}+\mathrm{X}_{\mathrm{de}}\left(s_{\mathrm{De}, \mathrm{s}}-s_{\mathrm{De}, \mathrm{f}}\right) \\
& \dot{m}_{p}=\rho_{D e} A_{D e} V_{D e} \\
& \mathrm{~h}_{\mathrm{nt}}+\frac{1}{2} \mathrm{~V}_{\mathrm{nt}}^{2}=\mathrm{h}_{\mathrm{de}}+\frac{1}{2} \mathrm{~V}_{\mathrm{ne}}^{2} \\
& \mathrm{~h}_{\text {De }}=\mathrm{h}_{\text {Def }}+\mathrm{x}_{\text {de }}\left(\mathrm{h}_{\text {Des }}-\mathrm{h}_{\text {Def }}\right)
\end{aligned}
$$

$$
\frac{1}{\rho_{D C}}=\frac{1}{\rho_{D e, f}}+x_{D e}\left(\frac{1}{\rho_{\text {De }, g}}-\frac{1}{\rho_{\text {Deff }}}\right)
$$

$$
a_{D e}=\sqrt{\left(\frac{\partial P}{\partial \rho}\right)_{2}}
$$

Again, the pressure at the nozzle exit, $P_{n e}$, is assumed and the above system is solved by iteration until convergence.

\section{Secondary Flow:}

Since the secondary flow is all liquid, Bernoulli's equation can be applied between the inlet and exit of the secondary flow tube:

$$
P_{\infty}=P_{\infty}+\frac{1}{2} P_{\infty} V_{x}^{2}
$$

where it has been assumed that the liquid density is nearly constant throughout the secondary fluid path;

$$
\rho_{s e}-\rho_{s}
$$
path yields

The continuity equation in the secondary flow

$$
\dot{\mathrm{m}}_{s}=\Phi \dot{\mathrm{m}}_{\mathrm{p}}=\rho_{\mathrm{se}} \mathrm{A}_{\mathrm{se}} \mathrm{V}_{\mathrm{se}}
$$

where the entrainment ratio, $\Phi$, is known a priori. Equations (18) through (20) can thus be solved to produce the pressure and velocity at the secondary flow path exit, $P_{s e}$ and $V_{s e}$, respectively.

\section{Mixing Chamber:}

The underlying assumption in the mixing chamber is: $P_{m e}=P_{s e}=$ the pressure along the slanted sides. The continuity momentum, and energy equations in the mixing chamber are thus: 


$$
\begin{aligned}
& \dot{\mathrm{m}}_{\mathrm{p}}(1+\Phi)=\rho_{\mathrm{me}} \mathrm{A}_{\mathrm{de}} \mathrm{V}_{\mathrm{me}}(\mathrm{AL}) \\
& P_{n e} A_{x e}+P_{s e} A_{s e}-P_{m e} A_{m e}=\quad D x \\
& \dot{m}_{p}(1+\Phi) V_{m e}-\dot{m}_{p} V_{s e}-\dot{m}_{p} \Phi V_{s e} \\
& \left\{\mathrm{~h}_{\mathrm{De}}+\frac{1}{2} \mathrm{~V}_{\mathrm{se}}^{2}\right\}+\left\{\mathrm{h}_{\mathrm{se}}+\frac{1}{2} \mathrm{~V}_{\mathrm{se}}^{2}\right\} \Phi= \\
& \left.h_{\text {me }}+\frac{1}{2} V_{m e}^{2}\right\}(1+\Phi)
\end{aligned}
$$

Equations (21) through (23) along with the equations of state

$$
\begin{aligned}
& s_{\text {me }}=s_{\text {mef }}+x_{\text {me }}\left(s_{\text {mas }}-s_{\text {mes }}\right) \\
& h_{\text {me }}=h_{\text {mest }}+x_{\text {me }}\left(h_{\text {mess }}-h_{\text {meff }}\right) \\
& \frac{1}{\rho_{\text {me }}}=\frac{1}{\rho_{\text {mes }}}+x_{\text {me }}\left(\frac{1}{\rho_{\text {mes }}}-\frac{1}{\rho_{\text {meff }}}\right)
\end{aligned}
$$

and the equations defining the Mach number and the speed of sound

$$
\begin{aligned}
& M_{m e}=\frac{V_{m e}}{a_{m e}} \\
& a_{m e}=\sqrt{\left(\frac{\partial P}{\partial \rho}\right)},
\end{aligned}
$$

constitute a system capable of fully determining the state of the mixture at the exit of the mixing chamber. This includes $\rho_{m e}, V_{m e}, A_{m e}, h_{m e}, s_{m e}, M_{m e}$, and $a_{m e}$.

\section{Diffuser:}

The conditions at the mixing chamber exit were assumed identical to those at the diffuser inlet for purposes of the analysis presented in this paper. Before this analysis is performed, a test for the existence of shock waves and for whether the flow is subsonic or supersonic is carried out. The Mach number computed above determines the latter issue and in case it is larger than unity, a shock wave is assumed to occur and the following system of equations is assumed to apply:

$$
\begin{gathered}
\rho_{m e} V_{m e}=\rho_{s e} V_{s} \\
P_{m e}+\rho_{m e} V_{m e}^{2}=P_{s e}+\rho_{s} V_{s=}^{2} \\
h_{m e}+\frac{1}{2} V_{m e}^{2}=h_{m}+\frac{1}{2} V_{s}^{2} \\
\rho=\rho(P, h)
\end{gathered}
$$

Again, the pressure is assumed and an iterative solution is carried out until convergence is reached.

If, on the other hand, the Mach number at the mixing chamber exit is less than unity, a subsonic solution is assumed and an isentropic flow analysis similar to the one carried out previously is performed in the diffuser. This yields the conditions at the diffuser exit, $P_{d e}, V_{d e}, x_{d e}, h_{d k}, \rho_{d e}, M_{d e}$, and $a_{d e}$.

\section{RESULTS AND DISCUSSION}

The mathematical model presented in the previous section was solved numerically in order to determine the distribution of thermodynamic states. In addition, the area of the mixing chamber exit was calculated as part of the solution instead of being specified a priori. This was necessitated by the constant-pressure mixing assumption applied to the mixing chamber. The analysis of a variable-area, fixed-geometry ejector of this type would necessarily require a streamwise pressure gradient during the mixing process except at a single operating point. In other words, the results associated with the constantpressure mixing assumption are a series of design points as opposed to an analysis of an ejector of fixed geometry. Previous analyses of this type (Fairuzov and Bredikhin, 1995) have attempted to analyze fixedgeometry ejectors using a quasi constant-pressure model. This approach seems to create potential contradictions in simultaneously satisfying the constantpressure constraint and the conservation laws in the mixing chamber. 
Figure 2 displays the mixing chamber exit-todiffuser exit area ratio as a function of the entrainment ratio for nozzle inlet temperatures of $-5,0$, and $5^{\circ} \mathrm{C}$. As is evident, the area ratio is a rather complicated function of the entrainment ratio for specific primary and secondary fluid inlet states. This is primarily the case because of the competing effects of the monotonically increasing velocity and the relatively complicated variation in density. For a specified mass flow rate, the area varies inversely with the product of density and velocity, hence leading to the observed trends in Figure 2. The density itself varies due to the decreasing pressure in competition with the decreasing mixture quality, shown in Figure 3. The latter falls rather rapidly with the entrainment ratio in the low entrainment ratio regime, while it exhibits a minimum at higher values. This behavior is explained by the quadratic dependence of the mixing chamber pressure on the entrainment ratio. In the low entrainment ratio regime, the pressure varies little so that the observed reduction in quality is due to the effect of the low secondary fluid temperature. On the other hand, for higher entrainment ratios, the thermal effect becomes dominated by the rapidly decreasing pressure. The effect of the primary inlet temperature is thermal, similar to the effect of the secondary fluid in the low entrainment ratio regime. Thus, lower primary fluid temperatures are observed to uniformly produce lower quality.

Figure 4 shows the variation of the mixing chamber exit Mach number with respect to the entrainment ratio and primary fluid inlet temperature. Increasing the entrainment ratio increases the Mach number because of the lower mixing chamber pressure and the associated higher mixture velocity. While the present model is also capable of handling supersonic flow cases, in the present paper we present only subsonic and sonic cases. The effect of increasing the primary fluid temperature, which also implies an increase in the primary fluid pressure, is to create a more energetic primary jet entering the mixing chamber, hence increasing the mixture velocity. An interesting observation is seen in the low entrainment ratio regime where a minimum mixture velocity increases monotonically. In this region, the speed of sound increases more rapidly than does the velocity. As the primary fluid temperature approaches that of the secondary, the primary fluid may not be energetic enough to cause the mixture to reach sonic conditions. This trend is observed for the $-5^{\circ} \mathrm{C}$ primary fluid temperature case.

The diffuser decelerates the flow from the mixing chamber throat to the ejector exit, which is of constant area. Shown in.Figure 5 is the variation of the diffusers exit quality as a function of the entrainment ratio and primary fluid inlet temperature. There are competing physical effects of pressure and temperarure analogous to those described in Figure 3. In the low entrainment ratio regime, the thermal effect dominates the mild pressure effect, while the reverse is true in the high entrainment ratio regime. For all cases examined, the ejector exit quality remained above zero in contrast to some of the experimental observations repored by Fairuzov and Bredikhin (1995) where liquid was observed at the ejector exit under certain conditions. The constraints of the constant-pressure mixing, coupled with the choice of baseline fluid inlet states, selected a subset of possible experimental cases, none of which corresponded to one of those of Fairuzov and Bredikhin (1995).

Figure 6 shows the variation of the diffuser exit Mach number as a function of the entrainment ratio and the primary fluid inlet temperature. As entrainment ratio is increased, one observes a knee in the curve above which the exit Mach number rapidly becomes unmanageably high, especially with a low primary fluid temperature. In that case, very little diffusion is occuring because the throat size necessary to accommodate constant-pressure mixing approaches that of the diffuser exit. At higher primary fluid temperatures, a practical amount of diffusion is possible even with choked conditions at the mixing chamber exit.

The variation of the diffuser static and stagnation exit pressures as functions of the entrainment ratio and the primary fluid inlet temperature can be seen in Figures 7 and 8. Similar trends are obseried in which the pressure ratio decreases with increasing entrainment ratio, qualitatively similar to performance curves for fixed-geometry ejectors. As expected, for the low primary fluid temperature case, the compression ratio is greater than one only for very low entrainment ratios. On the other hand, the high primary fluid temperature case resulted in practical compression ratios over the entire range. 


\section{CONCLUSIONS}

This paper presented a design-oriented model for a jet pump employing a two-phase primary fluid inducing a secondary liquid having the same chemical composition. The model is capable of accounting for phase transformations due to compression, expansion, and mixing employing an isentropic homogenous expansion/compression method of analysis. For given system requirements for flow rate and inlet fluid states, the model predicts the required mixing chamber throat necessary to accomplish constant-pressure mixing. For practical cases, the required throat size increases with increasing entrainment ratio. Practical compression ratios can only be achieved with sufficiently energetic primary fluids. Similar to fixed-geometry ejectors, the variation of compression ratio with entrainment ratio follows a monotonically decreasing trend. The more energetic primary fluid appears to be more suitable for inducing high compression ratios than high entrainment ratios.

\section{ACKNOWLEDGEMENTS}

The primary author is indebted to the financial support provided by the Thermal and Life Support Division of the NASA-Marshall Space Flight Center. This work is partially based on a NASA/ASEE Summer Faculty Fellowship awarded to the primary author in 1996 and 1997 under Grant No. NASA-MSFC-NGT852819. The primary author is also indebted to the program Co-directors, Drs. G.R. Karr and L.M. Freeman. The authors would also like to acknowledge the support of the Department of Mechanical Engineering at the University of Florida and are especially indebted to Alice A. Jempson for skillfully typing the manuscript.

\section{REFERENCES}

'Anand, G., 1992, "Phenomenological and Mathematical Modeling of a High Pressure Steam Driven Jet Injector," Ph.D. Dissertation, The Ohio State University, Columbus, Ohio.

'Bredikhin, V.V., Gorbenko, G.A., Nikonov, A.A., and Fairuzov, Y.V., 1990, "Mathematical Modeling of Thermo-Circulating Loops with Jet Pumps," Hydrodinamic Processes in Multi-Phase Working Fluid Energy Plants, Kharkov Aviation Institute, Kharkov, Ukraine, pp. 3-10 (in Russian).
${ }^{3}$ Cunningham, R.G. and Dopkin, R.J., 1974, "Jet Breakup and the Mixing Throat Lengths for the Liquid Jet Pump," ASME Joumal of Fluids Engineering, Vol. 96, No. 3, pp. 216-226.

${ }^{4}$ Cunningham, R.G., 1995, "Liquid Jet Pumps for Two-Phase Flows," ASME Joumal of Fluids Engineering, Vol. 117, No. 2, pp. 309-316.

'Elger, D.F., McLam, E.T., and Taylor, S.J., 1991, "A New Way to Represent Jet Pump Performance," ASME Joumal of Fluids Engineering, Vol. 113, No. 3, pp. 439-444.

${ }^{6}$ Fairuzov, Y.V. and Bredikhin, V.V., 1995 , "Two-Phase Cooling System with a Jet Pump for Spacecraft," AIAA Journal of Thermophysics and Heat Transfer, Vol. 9, No. 2, April-June, pp. 285-291.

'Holladay, J.B. and Hunt, P.L., 1996, "Fabrication, Testing, and Analysis of a Flow Boiling Test Facility with Jet Pump and Enhanced Surface Capability," Research Proposal, NASA-Marshall Space Flight Center. Thermal and Life Support Division, Huntsville, Alabama.

${ }^{8}$ Holmes, H.R., Goepp, J., and Hewitt, H.W., 1987. "Development of the Lockheed Pumped Two Phase Thermal Bus," AlAA Paper 87-1626, June.

9Jiao, B., Blais, R.N., and Schmidt, Z., 1990, "Efficiency and Pressure Recovery in Hydraulic Jet Pumping of Two-Phase Gas/Liquid Mixtures," SPE Production Engineering, Vol. 5, No. 4, pp. 361-364.

${ }^{10}$ Marini, M., Massardo, A., Satta, A., and Geraci, M., 1992, "Low Area Ratio Aircraft Fuel JetPump Performance with and without Cavitation," ASME Joumal of Fluids Engineering, Vol. 114, No. 4, pp. 626-631.

"Neve, R.S., 1991, "Diffuser Performance in Two-Phase Jet Pump," International Joumal of Multiphase Flow, Vol. 17, No. 2, pp. 267-272. 


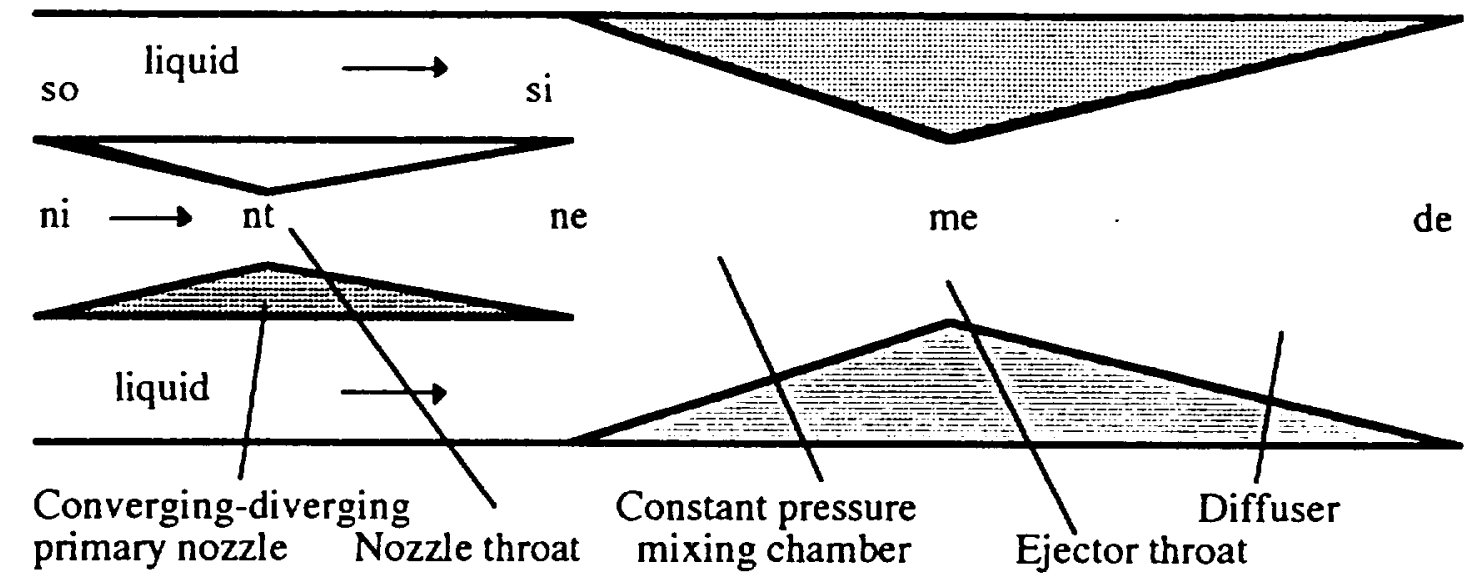

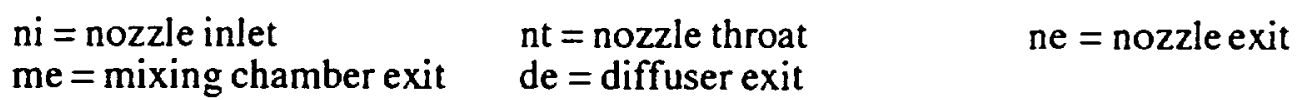

Figure 1. Schematic diagram of ejector with two-phase primary fluid and subcooled liquid secondary fluid.

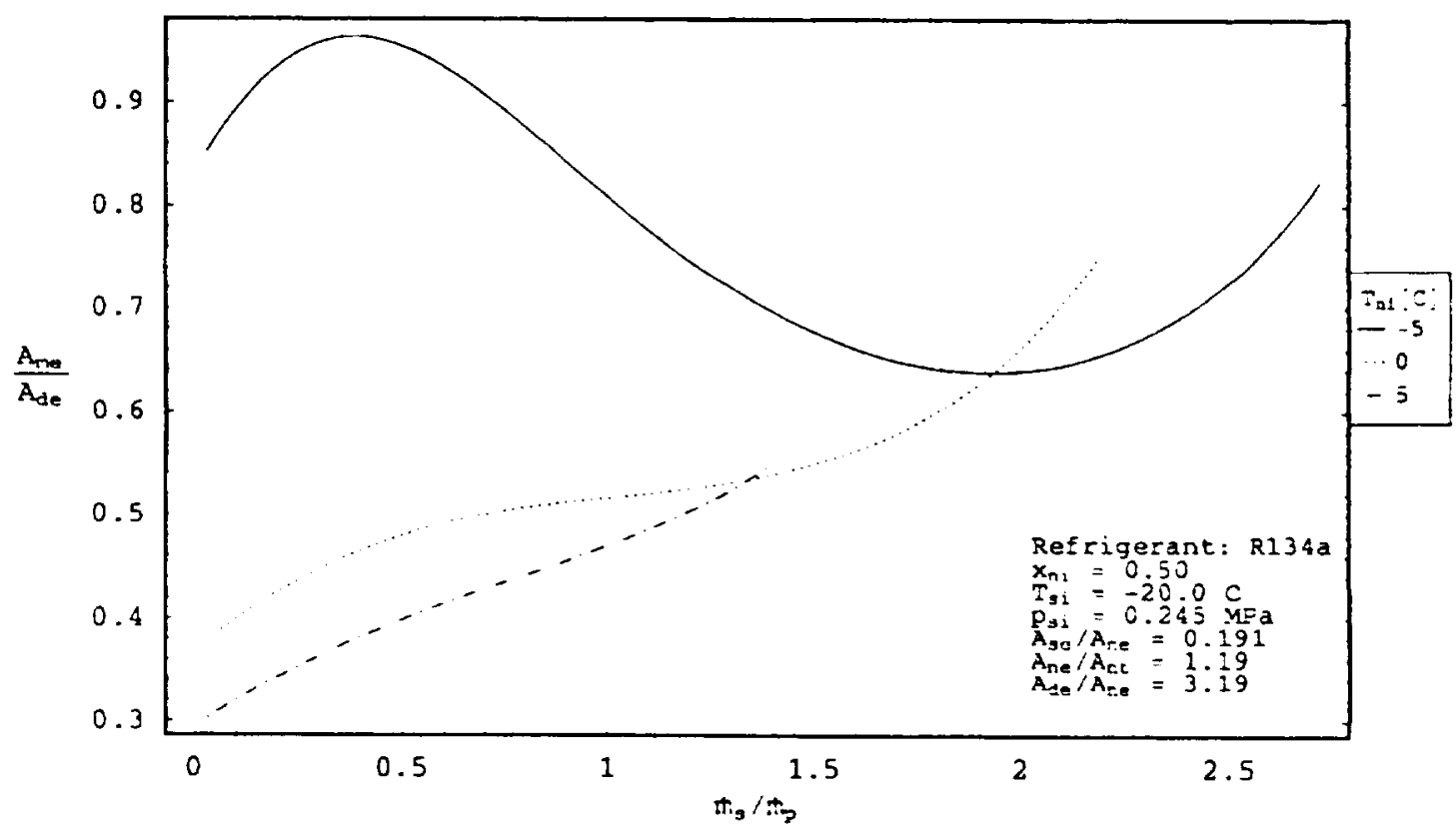

Figure 2. Mixing chamber throat to diffuser exit area ratio versus entrainment ratio for three values of the primary fluid inlet temperature. 


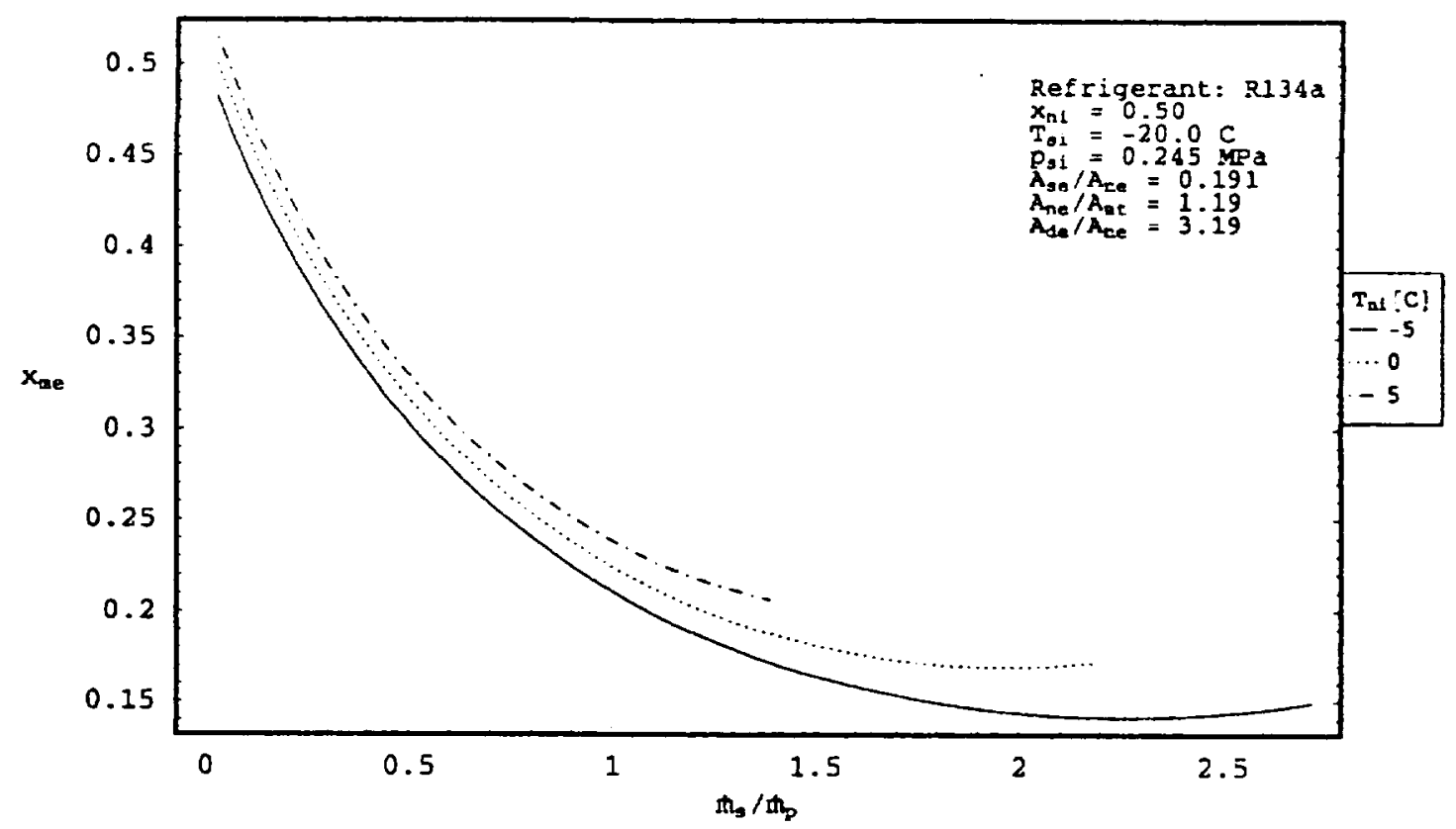

Figure 3. Mixing chamber exit quality versus entrainment ratio for three values of the primary fluid inlet temperature.

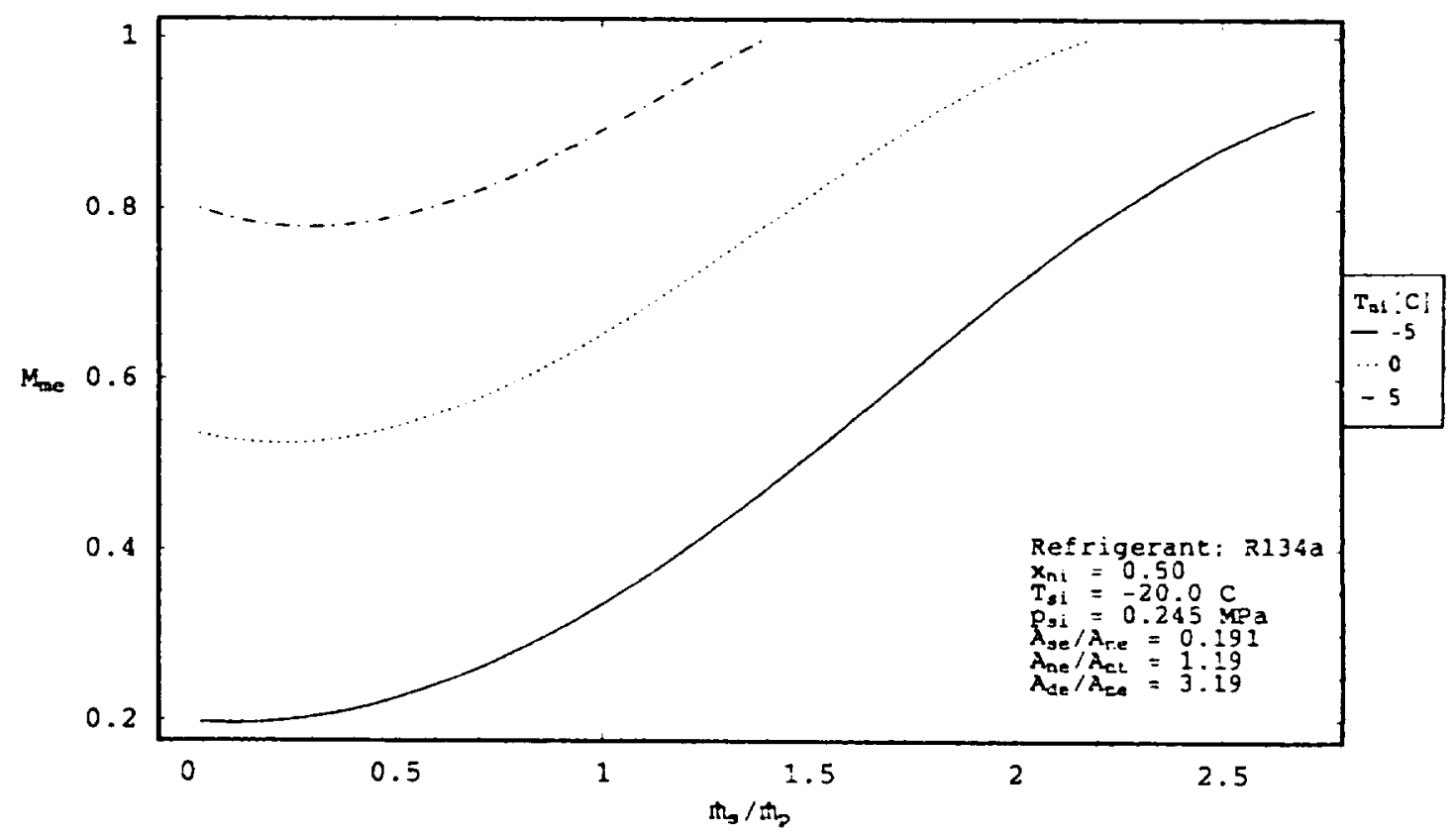

Figure 4. Mixing chamber exit Mach number versus entrainment ratio for three values of the primary fluid inlet temperature. 


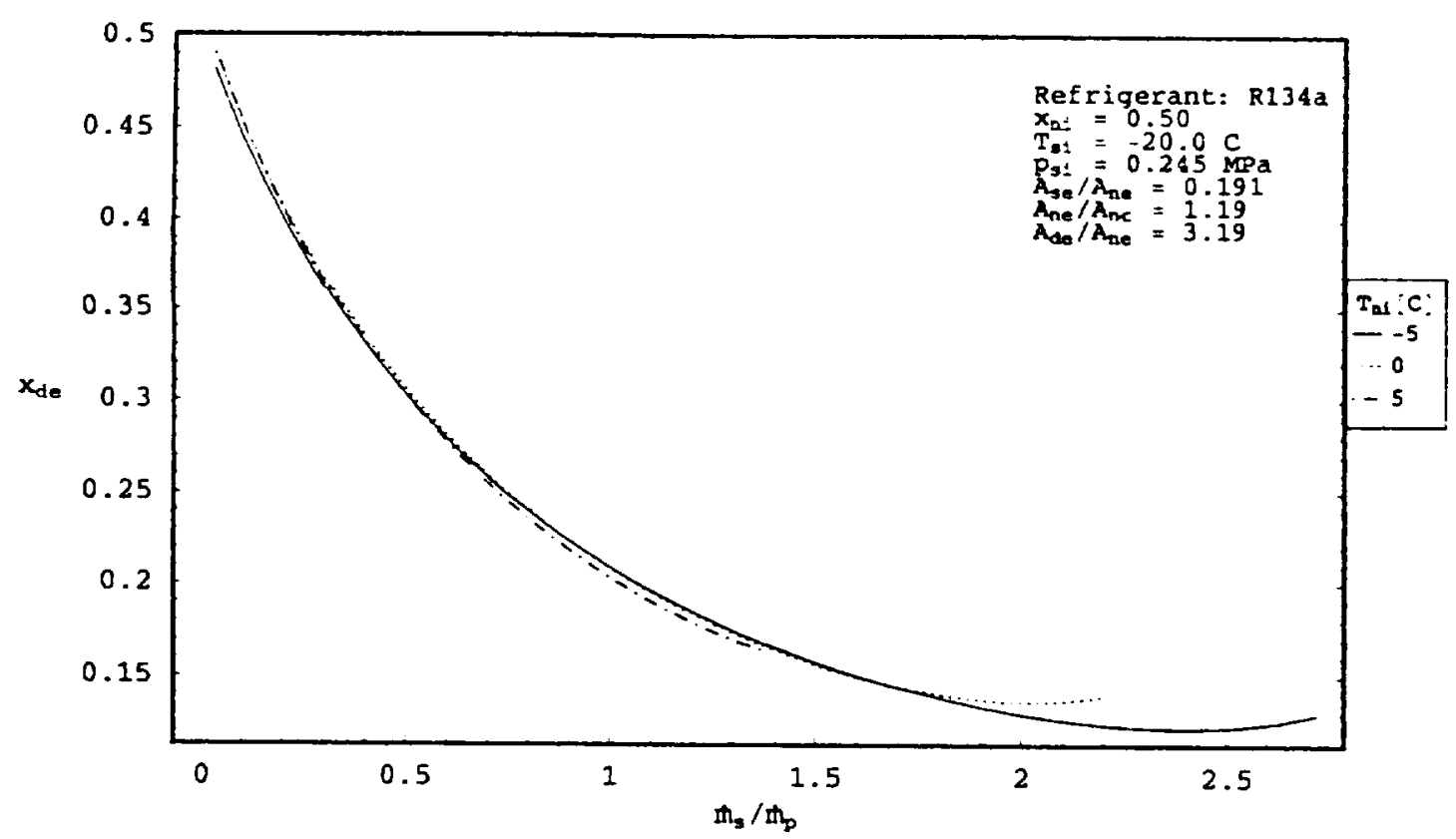

Figure 5. Diffuser exit quality versus entrainment ratio for three values of the primary fluid inlet temperature.

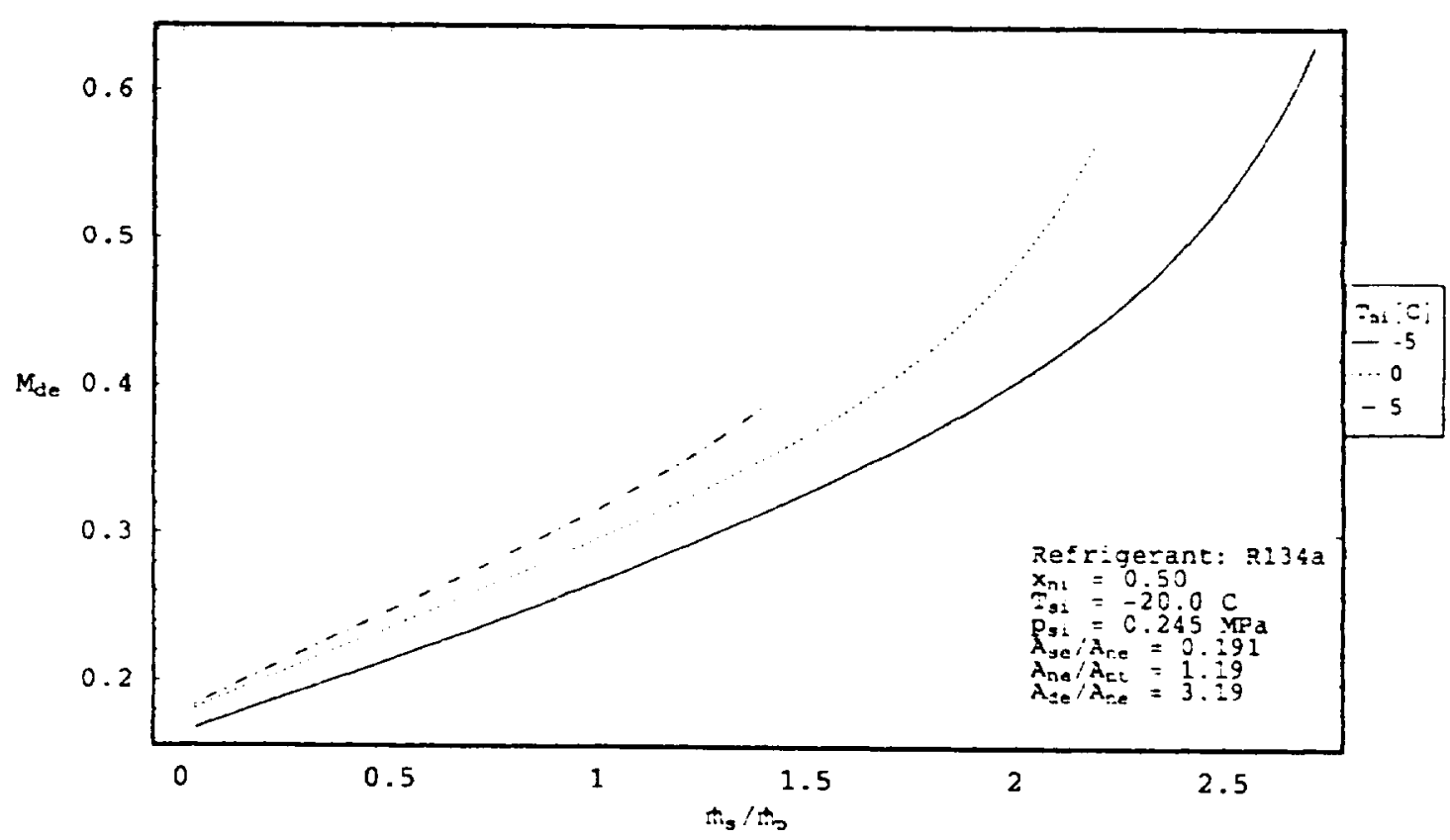

Figure 6. Diffuser exit Mach number versus entrainment ratio for three values of the primary fluid inlet temperature. 


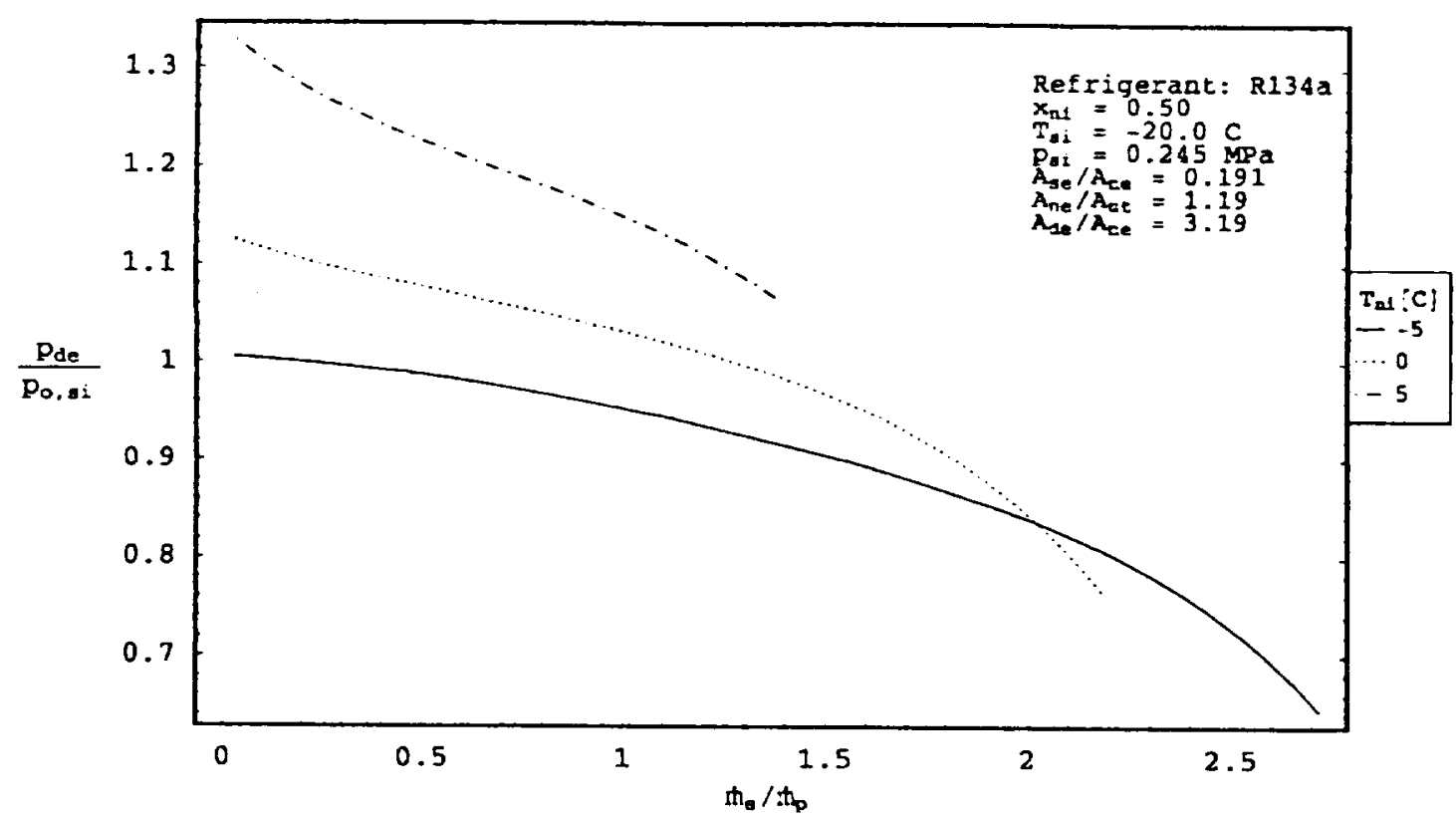

Figure 7. Ejector compression ratio based on exit static pressure versus entrainment ratio for three values of the primary fluid inlet temperature.

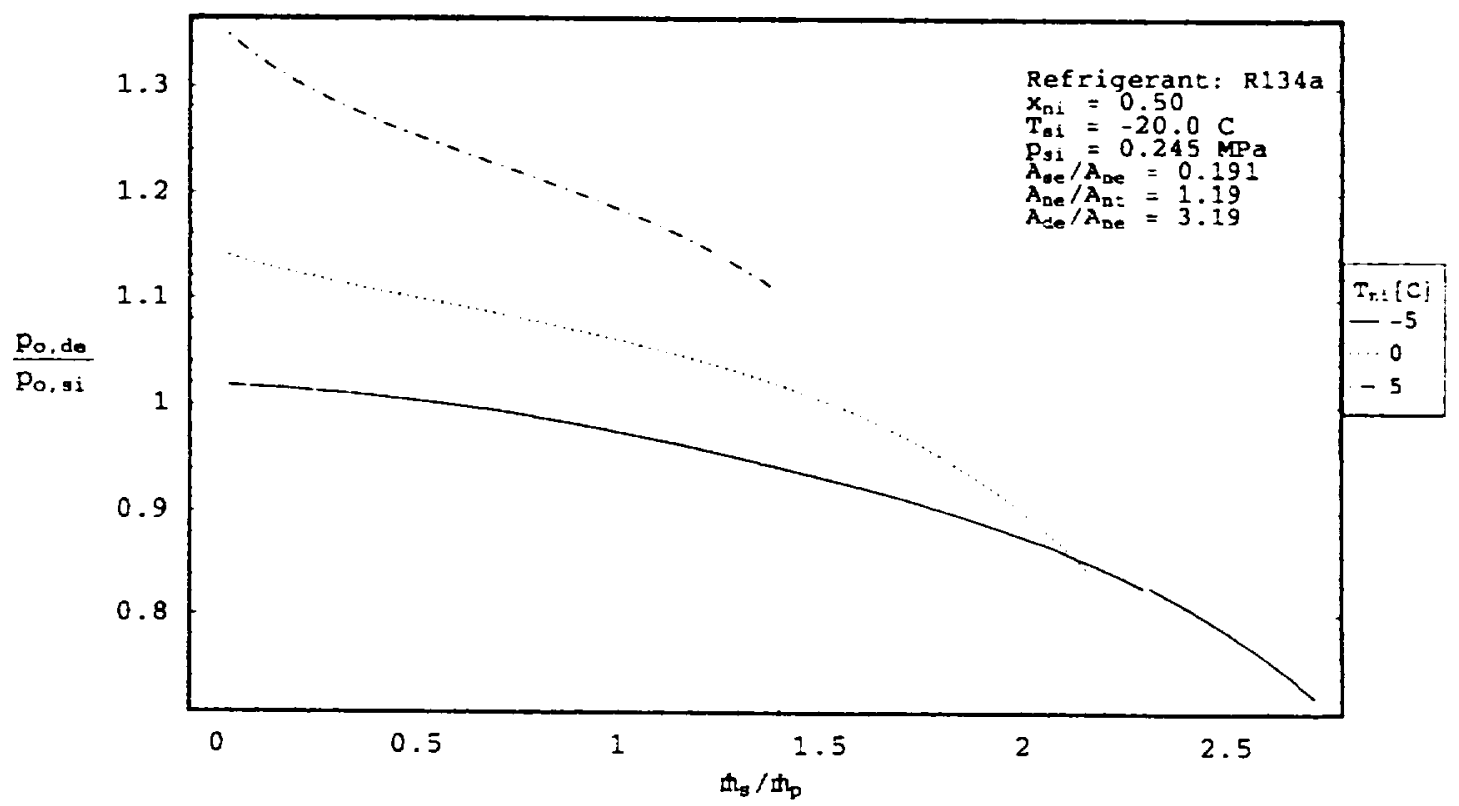

Figure 8. Ejector compression ratio based on exit stagnation pressure versus entrainment ratio for three values of the primary fluid inlet temperature. 